\title{
Spatiotemporal prediction of vancomycin-resistant Enterococcus colonisation
}

\author{
J. M. van Niekerk ${ }^{1,2,3^{*}}$, M. Lokate ${ }^{3}$, L. M. A. Braakman-Jansen ${ }^{1}$, J. E. W. C. van Gemert-Pijnen ${ }^{1}$ and A. Stein ${ }^{2}$
}

\begin{abstract}
Background: Vancomycin-resistant enterococci (VRE) is the cause of severe patient health and monetary burdens. Antibiotic use is a confounding effect to predict VRE in patients, but the antibiotic use of patients who may have frequented the same ward as the patient in question is often neglected. This study investigates how patient movements between hospital wards and their antibiotic use can explain the colonisation of patients with VRE.

Methods: Intrahospital patient movements, antibiotic use and PCR screening data were used from a hospital in the Netherlands. The PageRank algorithm was used to calculate two daily centrality measures based on the spatiotemporal graph to summarise the flow of patients and antibiotics at the ward level. A decision tree model was used to determine a simple set of rules to estimate the daily probability of patient VRE colonisation for each hospital ward. The model performance was improved using a random forest model and compared using 30\% test sample.
\end{abstract}

Results: Centrality covariates summarising the flow of patients and their antibiotic use between hospital wards can be used to predict the daily colonisation of VRE at the hospital ward level. The decision tree model produced a simple set of rules that can be used to determine the daily probability of patient VRE colonisation for each hospital ward. An acceptable area under the ROC curve (AUC) of 0.755 was achieved using the decision tree model and an excellent AUC of 0.883 by the random forest model on the test set. These results confirms that the random forest model performs better than a single decision tree for all levels of model sensitivity and specificity on data not used to estimate the models.

Conclusion: This study showed how the movements of patients inside hospitals and their use of antibiotics could predict the colonisation of patients with VRE at the ward level. Two daily centrality measures were proposed to summarise the flow of patients and antibiotics at the ward level. An early warning system for VRE can be developed to test and further develop infection prevention plans and outbreak strategies using these results.

Keywords: Vancomycin-resistant enterococci, Intrahospital patient movements, Spatiotemporal risk factors, Dynamic directed spatiotemporal graph, Centrality measure, Healthcare decision support

*Correspondence: j.m.vanniekerk@utwente.nl

${ }^{1}$ Department of Psychology, Health and Technology/Center for eHealth

Research and Disease Management, Faculty of Behavioural Sciences,

University of Twente, Enschede, The Netherlands

Full list of author information is available at the end of the article

\begin{abstract}
Background
Vancomycin-resistant enterococci (VRE) was first reported in Europe in 1986 [1] and since then has been the cause of severe health and monetary burdens [2]. The prevalence of VRE and VRE outbreaks have increased over the past 20 years in Europe [3]. Enterococcus faecalis and Enterococcus faecium are the Enterococci species
\end{abstract}

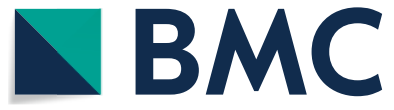

(c) The Author(s) 2022. Open Access This article is licensed under a Creative Commons Attribution 4.0 International License, which permits use, sharing, adaptation, distribution and reproduction in any medium or format, as long as you give appropriate credit to the original author(s) and the source, provide a link to the Creative Commons licence, and indicate if changes were made. The images or other third party material in this article are included in the article's Creative Commons licence, unless indicated otherwise in a credit line to the material. If material is not included in the article's Creative Commons licence and your intended use is not permitted by statutory regulation or exceeds the permitted use, you will need to obtain permission directly from the copyright holder. To view a copy of this licence, visit http://creativecommons.org/licenses/by/4.0/. The Creative Commons Public Domain Dedication waiver (http://creativeco $\mathrm{mmons}$.org/publicdomain/zero/1.0/) applies to the data made available in this article, unless otherwise stated in a credit line to the data. 
typically found in humans' gastrointestinal tracts, which could lead to bacteraemia, endocarditis, intra-abdominal and pelvic infections and urinary tract infections [1]. Patients are more than twice as likely to die from bloodstream infections caused by VRE as compared to a susceptible strain of Enterococcus [4]. Enterococci have properties that make them naturally resistant to the most used antimicrobial, and in particular, they can quickly become resistant to any new last-resort antimicrobials introduced.

Enterococci can survive on hospital surfaces and spread between patients and healthcare workers ( $\mathrm{HCW}$ ) using hands and surfaces as vectors [5]. In addition to direct patient-patient and HCW-HCW transmission pathways, there are five main transmission pathways for VRE inside a hospital: (1) patient to HCW; (2) patient to the environment; (3) HCW to patient; (4) environment to patient; (5) environment to HCW [6]. Since the VRE can survive on dry environmental surfaces for months, it could be a constant source for new outbreaks [7]. These reservoirs may persist despite routine cleaning procedures [8].

The immediate surroundings of a patient with VRE are likely to contain VRE reservoirs [9] and the odds of a patient being colonised with VRE increase when prior room occupants had VRE $[10,11]$. The risk of colonisation increases as the number and proportion of patients with VRE in the same unit increases [12]. Patients also face increased odds of VRE colonisation the more days they spend hospitalised [13]. Antibiotic use and immunosuppressing comorbidities such as leukaemia have been identified as risk factors for VRE colonisation $[4,13]$.

When a VRE outbreak occurs in a hospital, colonised patients are isolated, the extent of the outbreak is estimated and additional control measures are implemented if necessary [3]. Estimating the extent of an outbreak involves determining the contact group, usually at the ward level. The contact group consists of the patients who could potentially have been colonised during the outbreak. Contact tracing is typically used to determine the patients at risk. To verify which patients were indeed colonised, a screening process can be carried out, which can be expensive an uncomfortable for patients [14]. The benefits of improving the estimation accuracy of these contact groups are: (1) control measures are more effective, which translates into fewer transmissions and ultimately less infections; (2) fewer patients are burdened by the screening process; (3) less testing reduces the financial burden.

Even though estimation of the extent of an outbreak plays a critical role in outbreak management, few studies have investigated the relationship between the patient movements between hospital wards and the spread of microorganisms. Reasons for patients to move from one department to the other include deterioration of health; surgery after which they are moved to intensive care and afterwards to general care or more specialised care department; hospital logistics due to limited capacity. One study used centrality measures of intrahospital patient movements to predict the onset of clostridium difficile at the ward level [15]. The centrality of hospital antibiotic use, however, was not considered. Clostridium difficile can survive on hospital surfaces and patients are at risk from environmental vectors. Recent studies have shown that each intrahospital transfer increases a patient's odds of contracting clostridium difficile by $7 \%$ (95\% CI 1.02-1.13). To our knowledge, no similar studies exist for the VRE.

The effects of intrahospital patient movements and antibiotic usage in hospitals are usually studied separately in antimicrobial resistance (AMR) research. The use of antibiotics is usually included as a possible confounding effect to predict VRE colonisation in patients, but the use of antibiotics of other patients who may have frequented the same ward as the patient in question is often neglected. Hospitals are dynamic systems with many moving objects and each of those objects has a surface that can act as a vector for VRE. Furthermore, antibiotic use can increase the number of VRE in patients due to selection pressure which can then spread between patients $[8,16]$. For these reasons, VRE should be studied using covariates which include spatiotemporal movements of patients and antibiotics in the hospital.

This study investigates how patient movements between hospital wards and their antibiotic use can explain the colonisation of patients with VRE. We estimate the probability of a patient being colonised with VRE at the ward level using intrahospital movement data and antibiotic usage data. We estimate this probability using a decision tree model and a random forest ensemble model and compare the model performance as a sub-objective. This study is important because it allows infection prevention and control specialists and outbreak management staff to determine which wards are at risk of a VRE outbreak using commonly available hospital data.

\section{Methods}

\section{Patient movement and antibiotic data}

We used retrospective patient movement data from the University Medical Center Groningen (UMCG), one of the largest hospitals in the Netherlands with more than 10,000 employees and almost 1400 beds. Antibiotic usage and patient movement data are stored in an electronic health record (EHR) database. The period under study is January 2018 until December 2019. The anonymised data consist of admission and discharge 
dates for each department within the hospital and antibiotic administration times during admission. These data were used to calculate two covariates for each day during the period of study: (1) the number of patients in each ward (pat_num); (2) the number of patients using antibiotics in each ward (pat_num_ant).

\section{Spatiotemporal graph}

The intrahospital patient movements data can be used to construct a dynamic directed spatiotemporal graph (DG) [17]. The graph nodes are the wards and the edges between the nodes are the patients moving between the wards. The DG is spatiotemporal and dynamic since it presents the location of patients using a node structure over time. We created two DGs using the patient movement data and the antibiotics data. The first graph includes all patient movement between all wards. The second graph only includes the movements of patients using antibiotics.

\section{PageRank algorithm}

The PageRank (PR) algorithm aims to determine the centrality or "importance" of nodes given the number of other "important" nodes with vectors directed towards it [18]. In the context of this study, the PR algorithm estimates the probability distribution of an arbitrary patient ending up in a particular ward. We calculated the daily
PageRank probabilities for both DGs using a 30-day rolling time window: (1) PageRank of patient movements between wards (PR_pat_num) and (2) PageRank of patient movements currently using antibiotics (PR pat_num_ant). The PR_pat_num and PR_pat_num_ant represent the centrality of wards in terms of patients and antibiotics, respectively.

\section{VRE screening data}

The number of VRE tests fluctuated between 100 to 300 per week during the study period. A polymerase chain reaction (PCR) test was performed on rectal swabs from patients. If the PCR was suspected for VRE, culturing was performed. A patient was considered to be colonised with VRE if VRE could be isolated from culturing. Next Generation Sequencing (NGS) was performed on the VRE isolates and a minimum spanning tree based on the cgMLST was used to analyse the NGS sequence data for molecular epidemiological investigation.

All patients directly transferring from other hospitals were tested for VRE on admission over the entire study period. In addition, intensive care units (ICU) were screened for VRE twice a week. A VRE outbreak occurred at UMCG during the second half of 2018 (Fig. 1). The outbreak ward was screened 2-3 times a week during the outbreak period. Additional tests for VRE colonisation were performed during this period based on contact

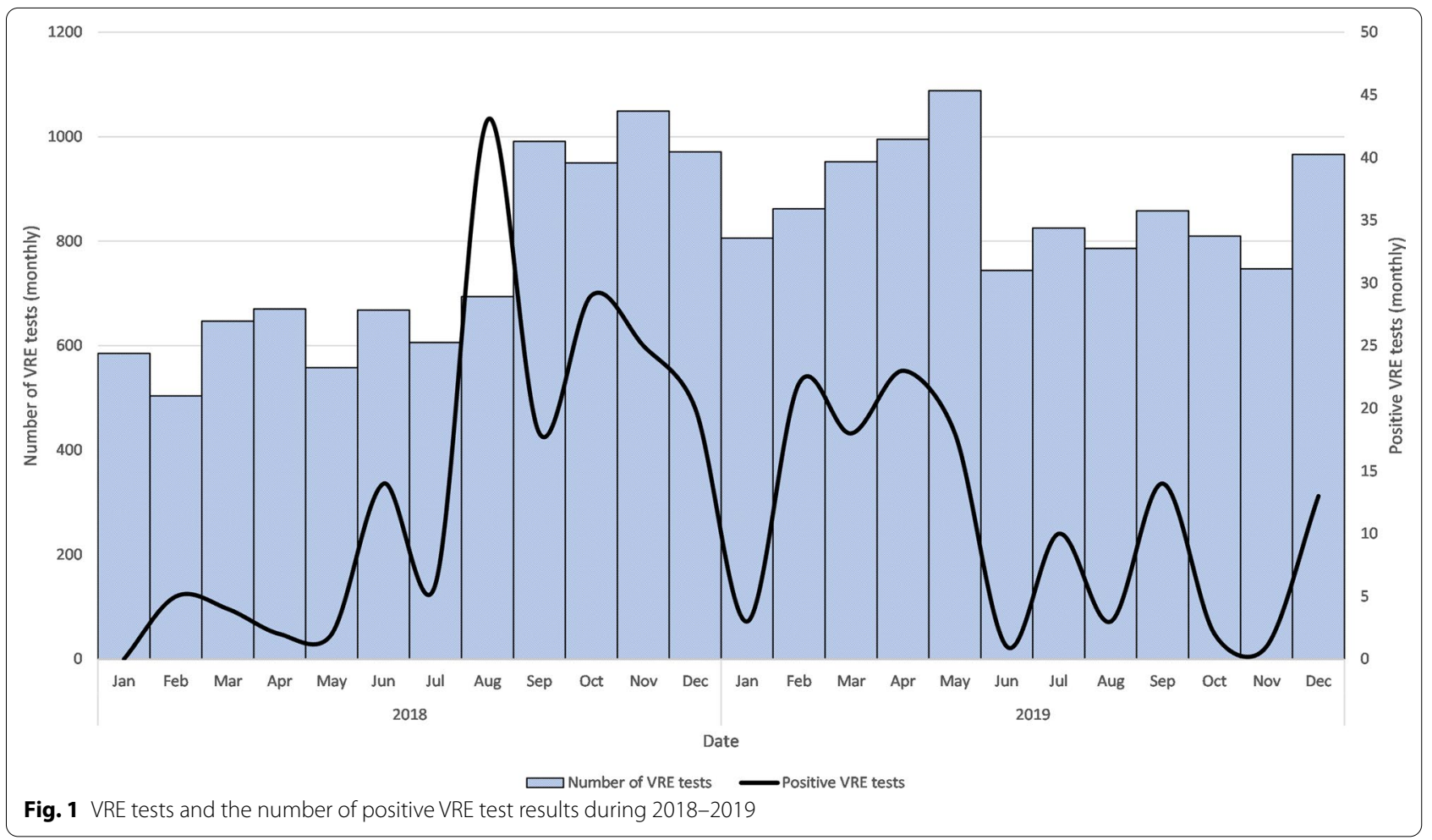


patients and wards closely related to the outbreak ward. From January 2019 onwards, patients admitted to the ward for at least ten days were tested every two weeks for VRE colonisation.

Additional infection prevention measures were taken during the VRE outbreak period. These include:

- Creating a cohort of positive patients and patients at risk of VRE. Nurses that work on the VRE-cohort do not work on the non VRE-cohort. Additional HCWs were used for night shifts to prevent nurses from working on both cohorts.

- More attention was given to hand hygiene measures. However, no measurements on compliance rates were performed during the outbreak.

- Additional use protective equipment like gloves and aprons were used for all patients.

- Intensified screening on VRE. Patients in the outbreak ward were screened three times per week. Admission screening and discharge screening for all patients.

- During a short period during the outbreak, the admission of new patients was completely halted.

- Contact tracing and screening of discharged patients, including screening at home.

- Intensified cleaning and even closure of a ward and moving patient to a secondary ward to clean the original ward.

- Environmental screening for VRE.

Between July-December 2018, 141 positive VRE tests were reported, with a peak of 25 positive tests in one week. In total, 48 patients tested positive for VRE over the study period.

\section{Modelling}

The binary outcome variable $Y$ was defined (1) and calculated using the VRE screening data over the study period.

$$
Y= \begin{cases}1, & \text { number of patients colonised with VRE in ward }>0 \\ 0, & \text { otherwise }\end{cases}
$$

We estimated the conditional probability that there is at least one patient colonised with VRE in a specific ward (Y) given the covariates pat_num, pat_num_ant, PR_pat_ num and PR_pat_num_ant (2). probability of $\mathrm{Y}$ [19]. The decision tree was grown using a $70 \%$ random training sample of the complete set of data. The data were split incrementally by adding question nodes. The question nodes consider the ability of each covariate to discriminate between the observed binary outcomes and formulates the question using the one that can discriminate best [20]. We used the Gini index to quantify the discriminatory ability of each covariate at the question nodes [19]. Continuing in this way, a tree branch structure is created, leading to the final decision or leaves of the tree.

\section{Random forest}

The model performance of decision trees was improved by creating an ensemble of decision trees and using them in unison to predict the outcome variable [20]. We used the same $70 \%$ randomly sampled training samples used to train the decision tree model. To build the random forest (RF) model, 500 random samples with replacement (bootstrap sample) were drawn from the training data and two random outcome variables were used to build a decision tree for each of the bootstrap sample. The probability of $Y$ was determined by calculating the proportion of the 500 trees that predicted $Y=1$.

We compared the model performance of the decision tree and random forest models using the remaining 30\% data as a test sample. The area under the receiver operating characteristic curve (ROC) was used to measure model performance as it provides a holistic view of how well the model predicts the outcome variable for different levels of sensitivity and specificity [21]. An AUC between 0.7 and 0.8 is considered as acceptable and between 0.8 and 0.9 excellent [123].

\section{Software}

The $\mathrm{R}$ statistical programming language was used to perform the analyses in this study [22]. Graphs were created and evaluated using igraph [23]. The decision trees and random forest models were built using the rpart and randomForest packages [24, 25]. In addition, the tidyverse $\mathrm{R}$ package was used to clean and structure the data [26].

\section{Results}

In total, 48 distinct wards were occupied over the 730 days in the study period (2018-2019). Of the possible 35,040 observations, if all the wards were occupied every

$$
P\left(Y=1 \mid p a t \_n u m, p a t \_n u m \_a n t, P R \_p a t \_n u m, P M \_p a t \_n u m \_a n t\right)
$$

\section{Decision trees}

A decision tree was used to determine a simple set of rules based on the covariates to estimate the conditional day, only 31,649 observations were collected, of which 1377 (5.45\%) had at least one patient with VRE. 


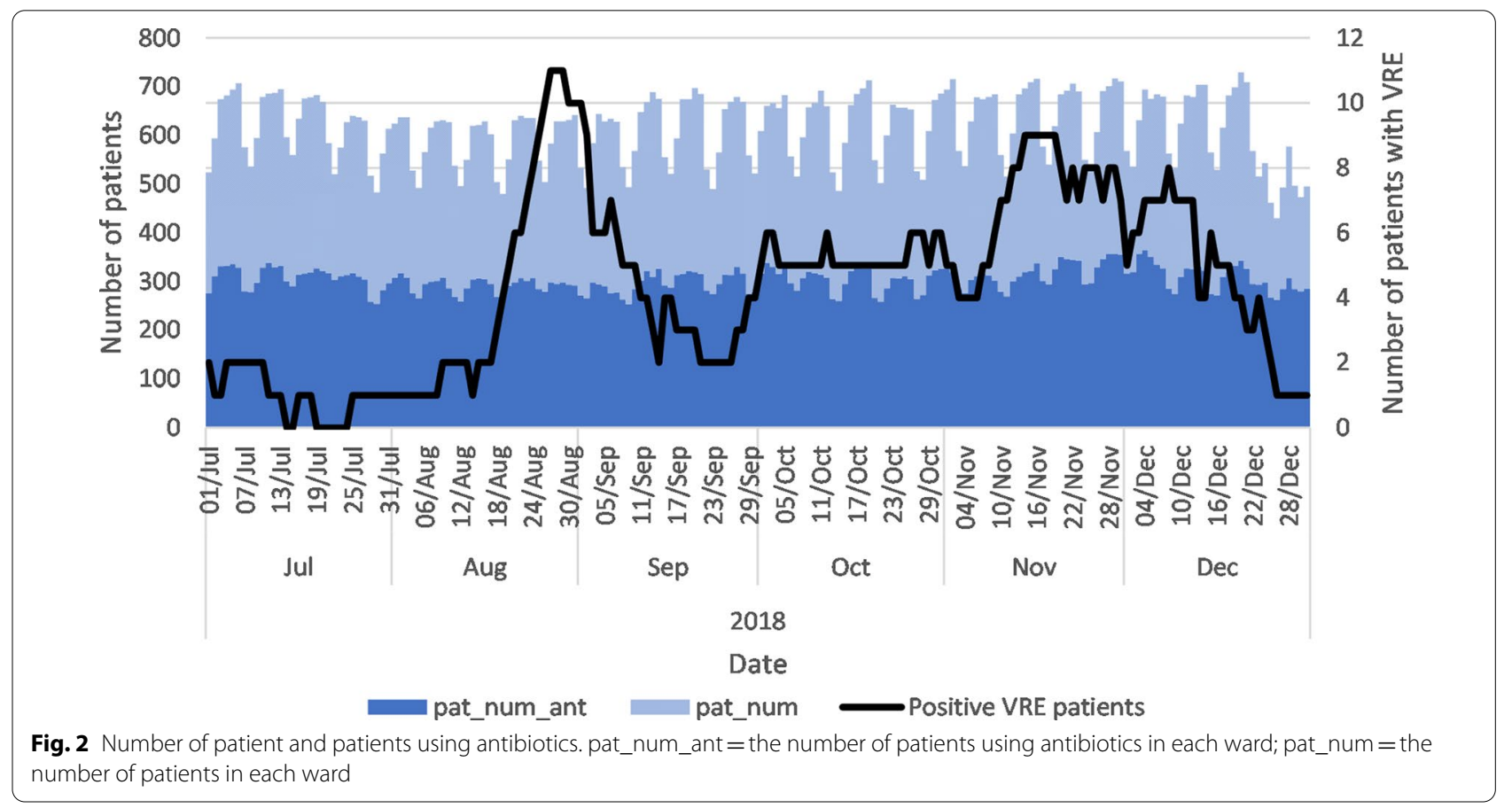

\section{Covariates}

The pat_num and pat_num_ant covariates are shown with the number of patients colonised with VRE during the VRE breakout period in 2018 in Fig. 2. We highlight the general care ward with many VRE colonised patients during this outbreak in Fig. 3. These results show a higher level of variation at the ward level, which conforms better to the number of patients colonised with VRE. The highest number of VRE colonised patients were observed in the last week of August 2018. At the hospital level, the

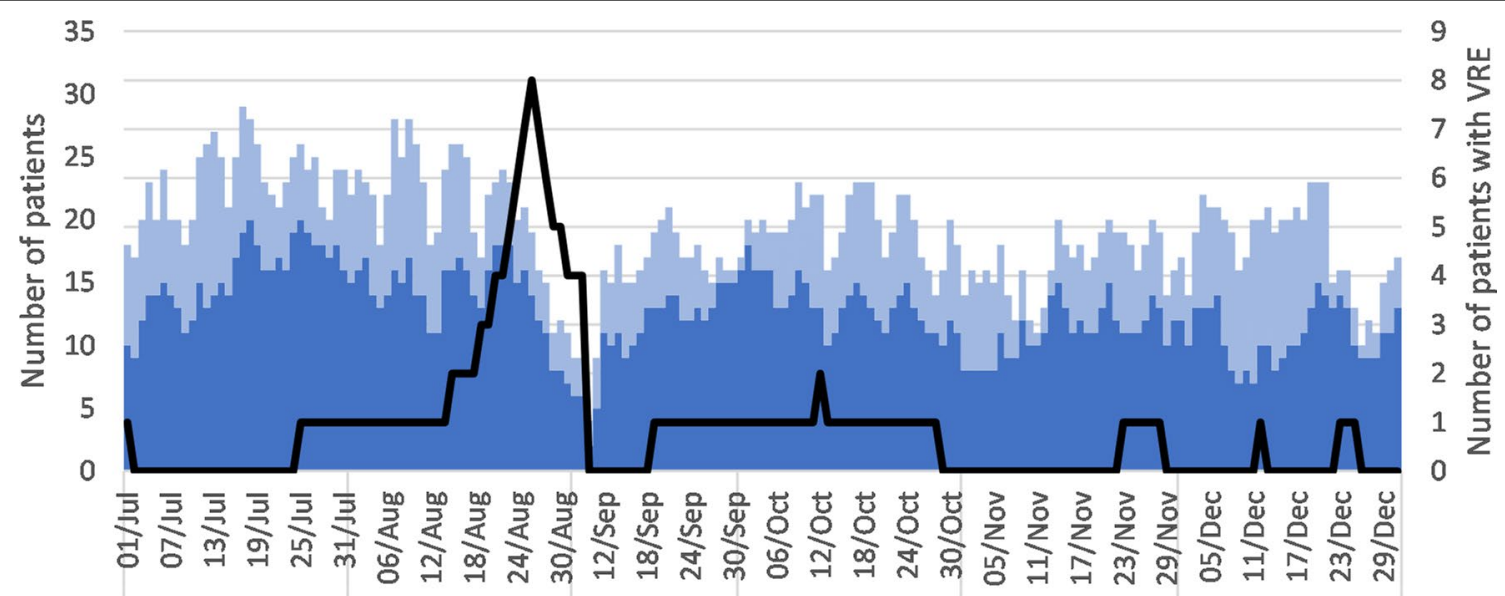

Jul

Aug

Sep

Oct

Nov

Dec

2018

Date

pat_num_ant_pat_num Positive VRE patients

Fig. 3 Number of patient and patients using antibiotics in example general care ward. pat_num_ant = the number of patients using antibiotics in each ward; pat_num $=$ the number of patients in each ward 
relationship between the pat_num_ant, pat_num and the number of VRE colonised patients is not evident. When the same data are shown at the ward level for the general care ward, these covariates are correlated with the number of VRE colonised patients.
Comparing the two PR_pat_num and PR_pat_num_ ant reveal that during this period, PR_pat_num_ant was higher than PR_pat_num (Fig. 4). This means that, on average, the probability of a patient using antibiotics to visit a ward was higher than for the total patient
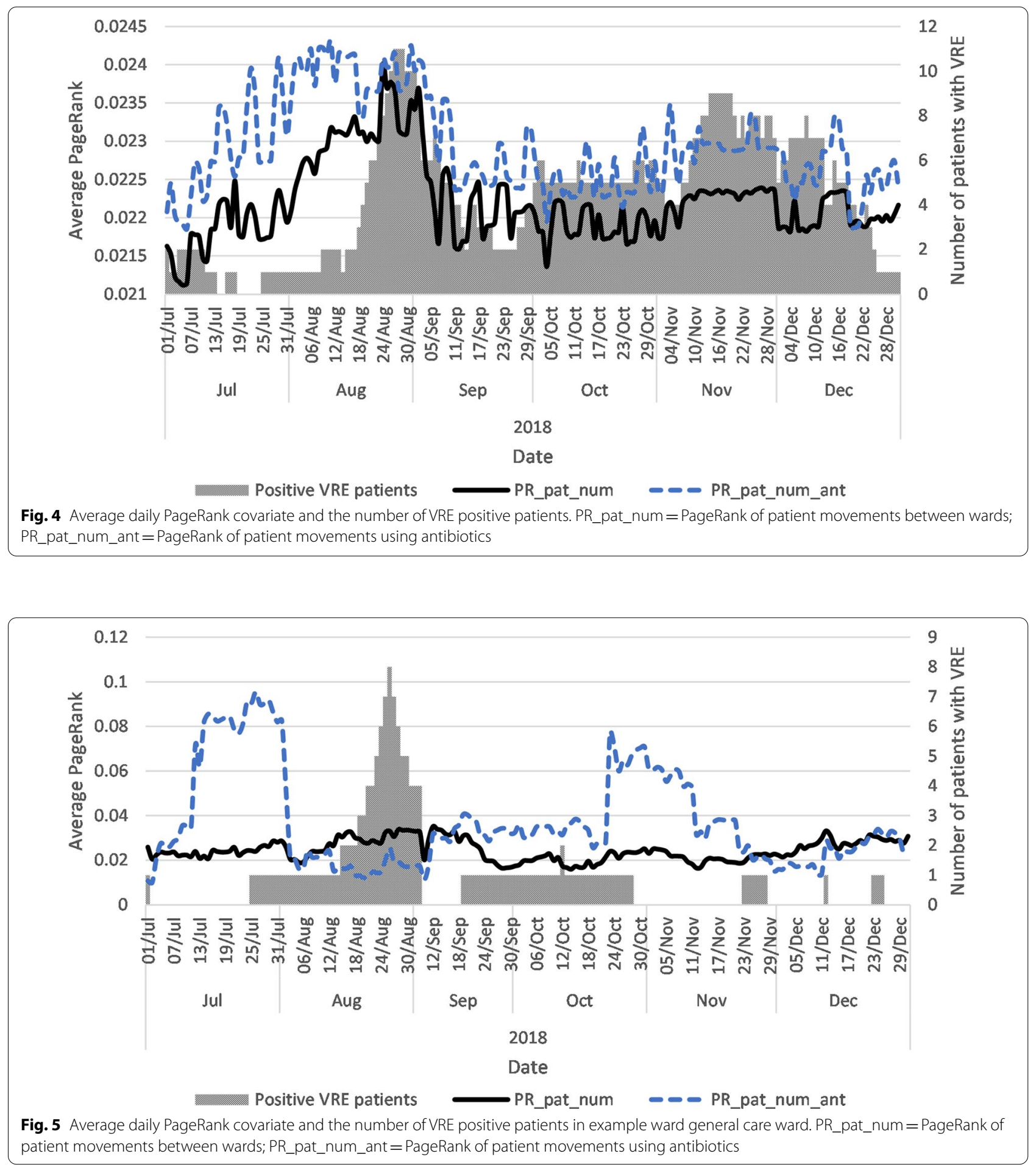
population. The same covariates are shown for the example general care ward in Fig. 5. The general care ward experienced a significant increase in PR_pat_num_ant during July and October 2018, which lasted for four weeks and yet PR_pat_num did not show a similar pattern. These results show that the two centrality covariates provide different information of the patient and antibiotics flow in a hospital at the ward level.

\section{Decision tree}

The $70 \%$ training sample had a $4.3 \%$ observations for which $Y=1$ at the root node (Fig. 6). The pat_num_ant the next node to consider the PT_pat_num_ant covariate. After dividing the training sample by the five nodes, we arrive at the seven leaves of the tree. The probabilities of the leave population range between $0.98 \%$ and $15.68 \%$. According to the order in which the covariates were used in the model, the pat_num_ant is the most important covariate to estimate the probability of a hospital ward having at least one patient colonised with VRE. The PR covariates are next in the order of importance to determine the final leaves of the tree. The decision tree results can be written and executed as a simple set of rules provided in (3).

$$
P(Y=1 \mid \text { pat_num, pat_num_ant, PR_pat_num, PM_pat_num_ant })=
$$

covariate splits the first nodes. If the number of patients is less than six, which is the case for $40 \%$ of the training sample, then there is a $0.098 \%$ probability that the ward has a VRE colonised patient. If the number of patients in a ward is six or more, but less than 13 , we continue to
0.0098 if pat_num_ant $<6$, 0.0326 if pat_num_ant $\in[6,13]$ AND PR_pat_num_ant $\in[0.022,0.029)$ AND PR_pat_num $\geq 0.025$, 0.0340 if pat_num_ant $\in[6,13]$ AND PR_pat_num_ant $<0.22$,

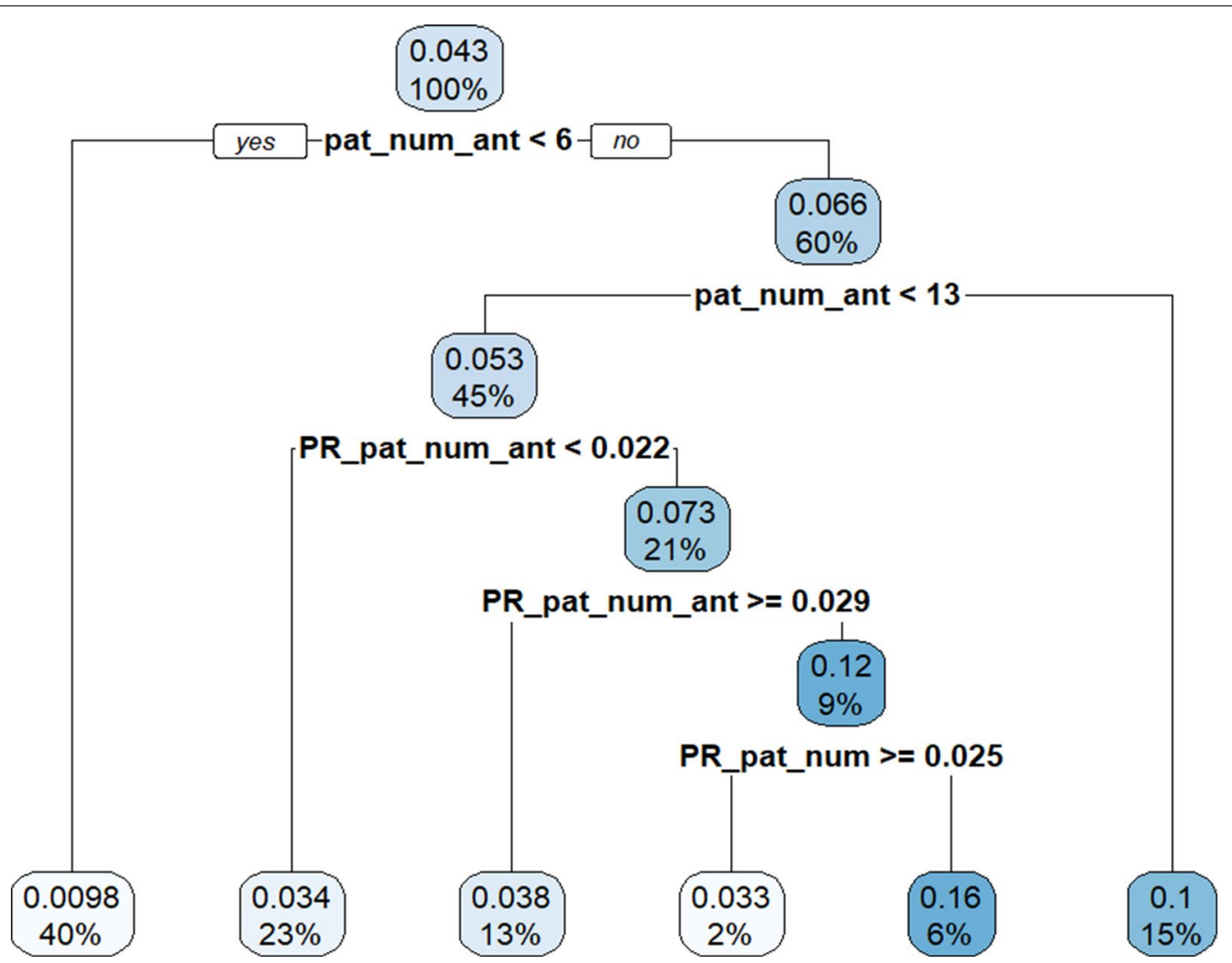

Fig. 6 Decision tree for the daily VRE colonisation in a hospital ward using PageRank and traditional covariates. pat_num_ant=the number of patients using antibiotics in each ward; PR_pat_num_ant = PageRank of patient movements currently using antibiotics; PR_pat_num = PageRank of patient movements between wards. In each node, the percentage of wards with at least one patient colonised with VRE is shown above the sample distribution of the node 


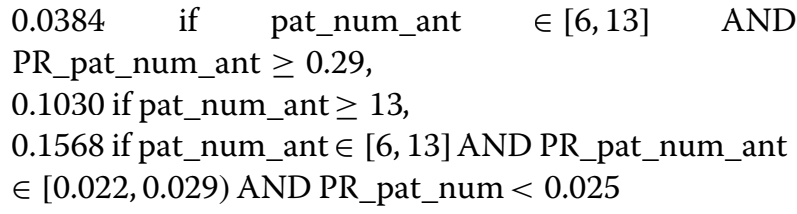

\section{Random forest}

The minimal depth provides insight into where a covariate occurs for the first time in the decision trees for the random forest and quantified variable importance. Covariates with lower minimal average depth are used to split larger proportions of the population due to higher discriminatory power. The results show that pat_num_ ant has the lowest average depth (0.61) and is most likely to be used in the root node. This result is consistent with our single decision tree model (Fig. 7). PR_pat_num was not used as a root node for any of the 500 decision trees. It has the largest average depth (1.93) in the trees, which means that it was generally used in nodes appearing lower in the decision trees.

We determined the covariate importance in the RF model by calculating the percentage increase in the mean square error (MSE) and the change in the residual sum of squares (RSS) of the model should random information replace the values of the model covariates. The results show that the PR covariates are the most important ones in terms of the MSE (Fig. 8) and RSS (Fig. 9) reductions.

\section{Model performance}

The performance of the models is compared to the Lorenz curves shown in Fig. 10. The Lorenz curve of the RF model is consistently higher than for the decision tree model. The RF model achieved an area under the curve of 0.883 and the decision tree model 0.755 on the $30 \%$ test set. This result confirms that the random forest model performs better than a single decision tree for all levels of model sensitivity and specificity on data not used to estimate the models. This is important to estimate the loss in model performance when choosing to use the simple set of rules produced by the decision tree model to calculate the probability of Y rather than using the RF model.

\section{Discussion}

This study showed how the movements of patients inside hospitals and their use of antibiotics could predict the VRE colonisation of patients at the hospital ward level. Two daily centrality measures were proposed to summarise the flow of patients and antibiotics at the ward level.

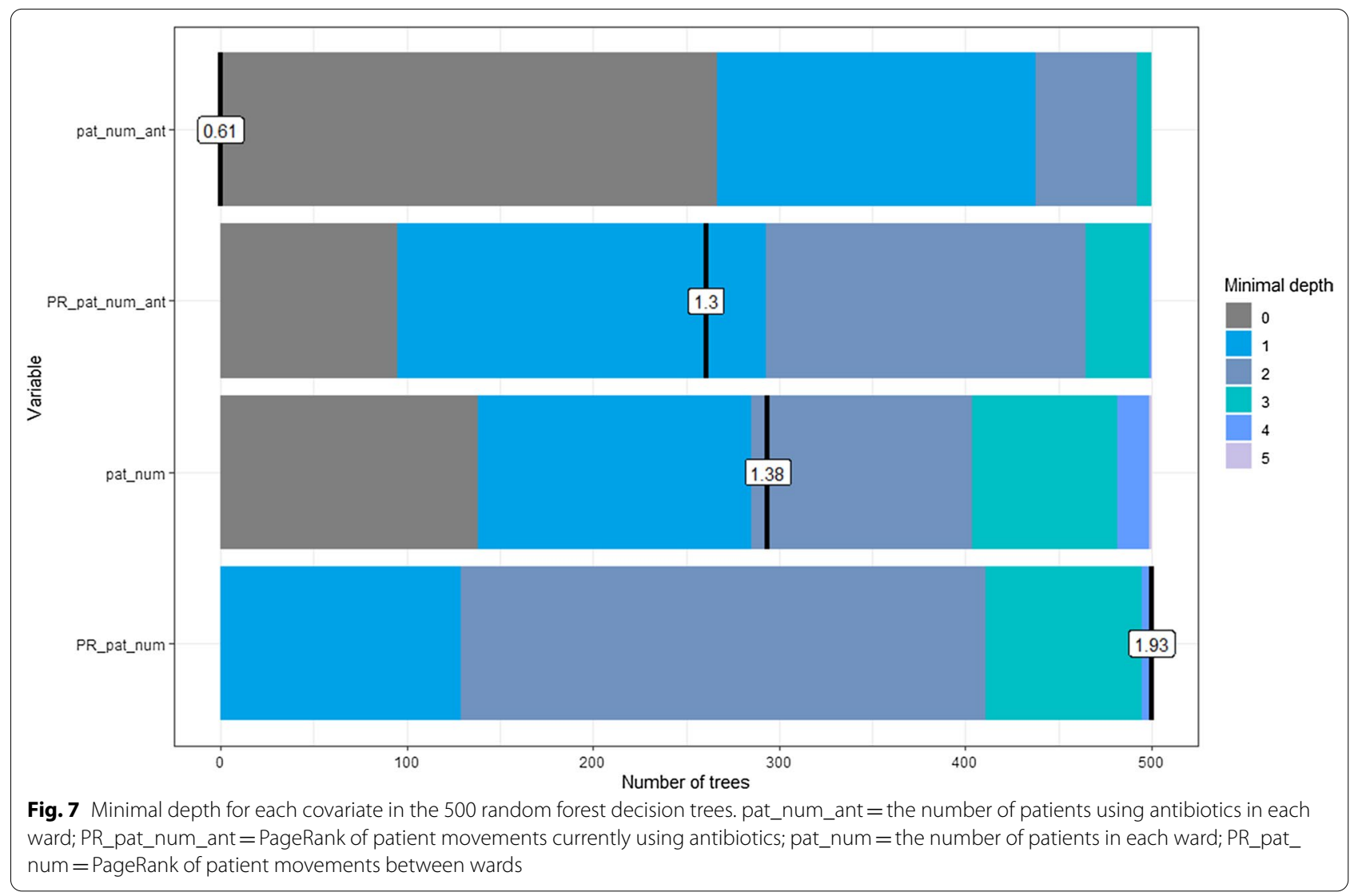




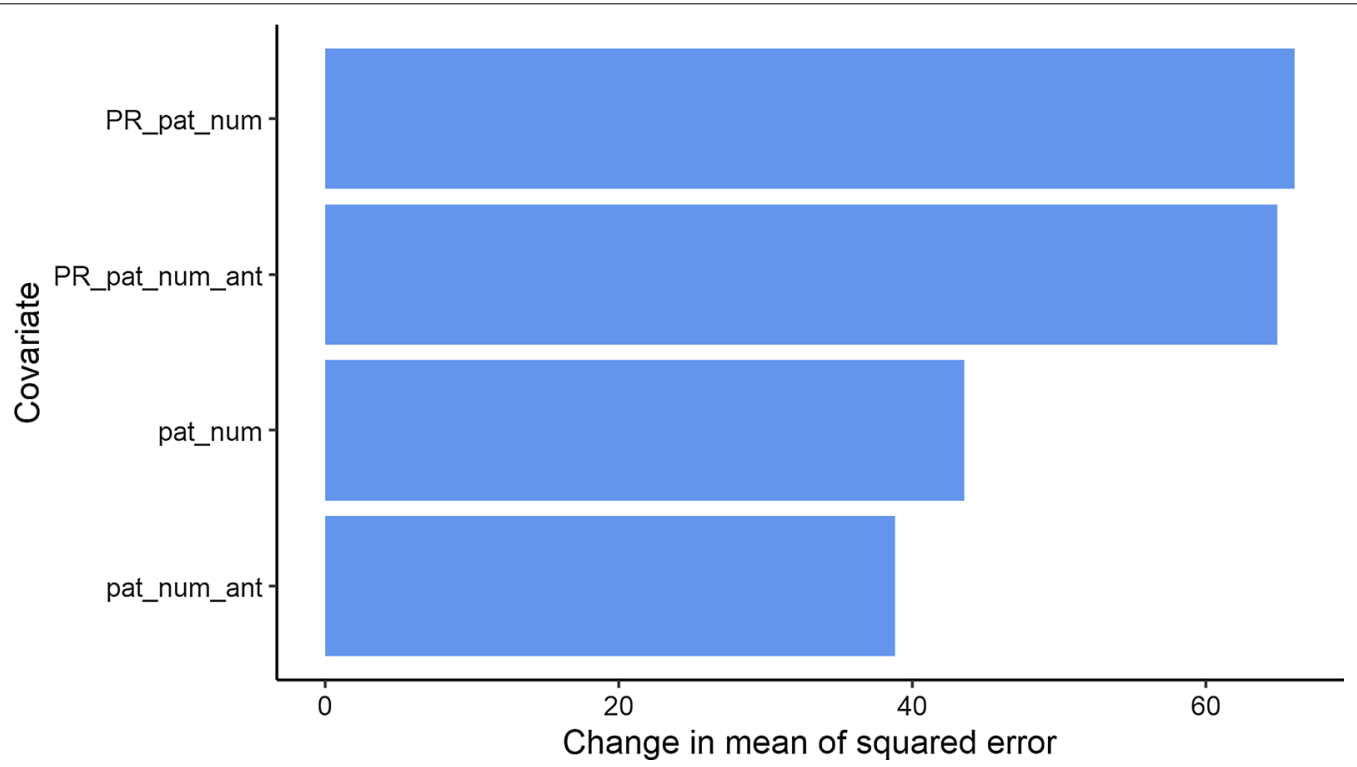

Fig. 8 The change in mean squared error when covariate values are replaced with random values. PR_pat_num=PageRank of patient movements between wards; PR_pat_num_ant = PageRank of patient movements currently using antibiotics; pat_num = the number of patients in each ward; pat_num_ant $=$ the number of patients using antibiotics in each ward

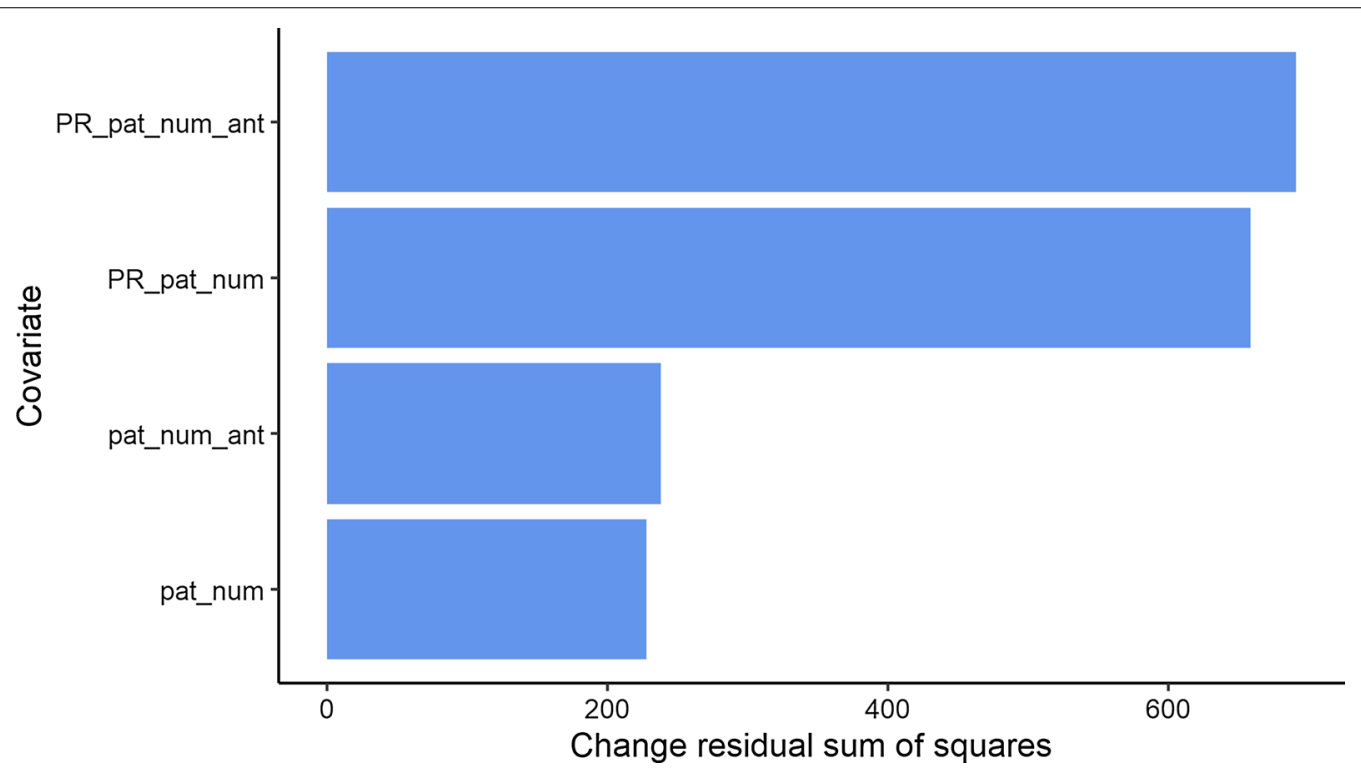

Fig. 9 The change in residual sum of squares when covariate values are replaced with random values. PR_pat_num_ant=PageRank of patient movements currently using antibiotics; PR_pat_num = PageRank of patient movements between wards; pat_num_ant=the number of patients using antibiotics in each ward; pat_num $=$ the number of patients in each ward

A simple set of rules were produced which can be used to monitor the risk of VRE colonisation in hospital wards. Using an ensemble method, a more accurate but more complicated model was developed, which can be applied to the same effect should resources allow for it.

The two PageRank covariates proposed offered new insight into the centrality of wards regarding patient and antibiotic movements and their interaction. This study used the covariates to predict VRE colonisation, but they can be used in many other studies concerning antimicrobial resistance in hospitals. Institutional surveillance monitors the usage of antibiotics but not the flow and concentration thereof. The proposed PR covariates can be used in conjunction with existing institutional 


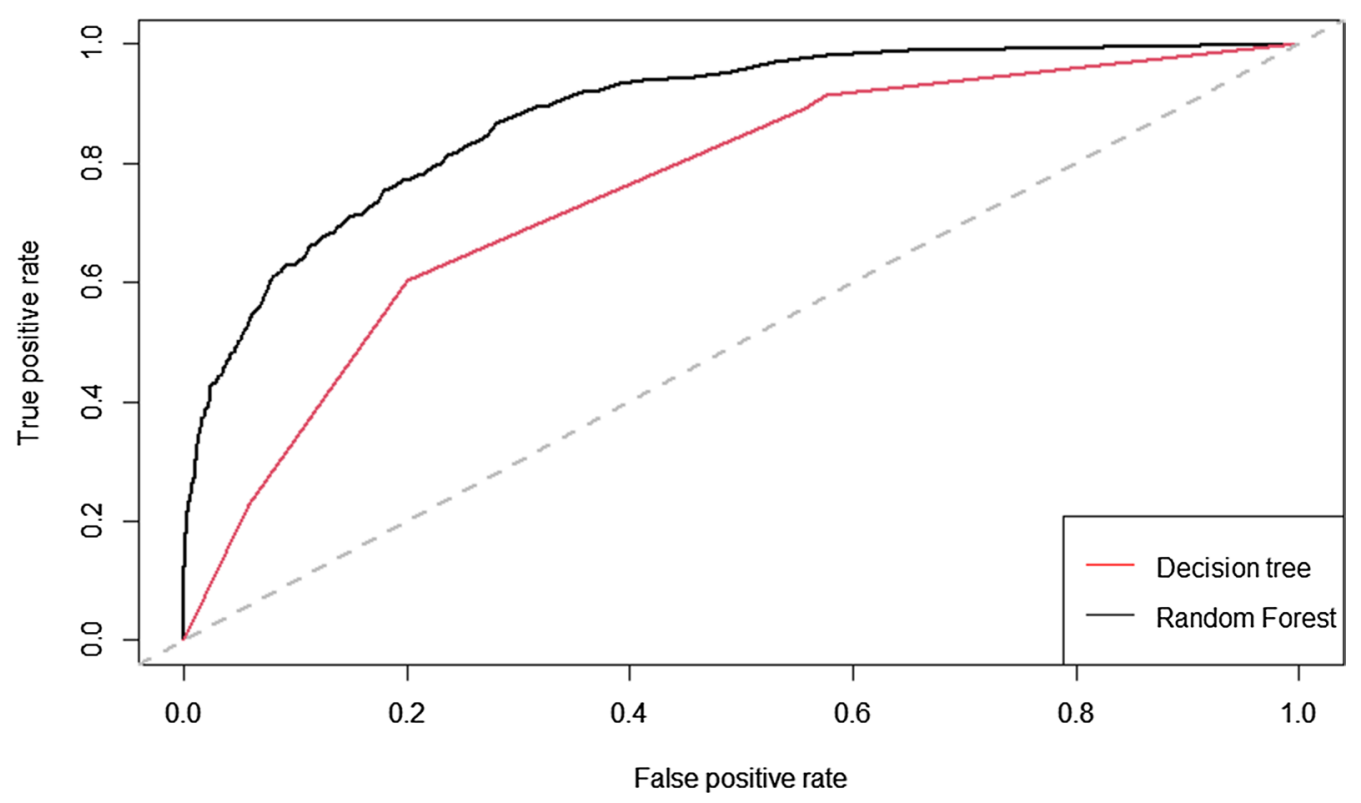

Fig. 10 Lorenz curves of the decision tree and random forest models

surveillance metrics to monitor the risks for VRE colonisation and AMR colonisation in general.

The decision tree model resulted in six simple questions and provided the probability that a ward has at least one patient colonised with VRE as an answer. This model enables hospitals to use passive data collected in their electronic health records to calculate this probability. To improve the accuracy of this model, a random forest model was built, which outperforms the decision tree model. The random forest model results were not as easily interpretable as that of the decision tree as it uses 500 smaller decision trees every time a probability is calculated. In practice, the model used will depend on the skills and resources of the hospital and its infection prevention and control specialists.

\section{Future work}

The results of this study can be used to develop an early warning system for VRE colonisation and other microorganisms with similar transmission mechanisms. The probabilities produced by the models presented can be used to classify the predicted VRE colonisation outcome according to the desired level of sensitivity and specificity for such a system. The results can then be updated daily or as frequently as the covariates can be calculated and evaluated by the infection prevention specialists to decide on the best course of action.

Our results showed that the value of the patient movement and antibiotic PR covariates sometimes move in the opposite direction over time. This divergence suggests that the proportion of patients using antibiotics changes over time. These covariates can be used together to determine if emerging divergences increase the risk of VRE colonisation.

\section{Limitation}

The study period was limited by the amount of data available for intrahospital patient movement, antibiotic use and VRE colonisation screening. UMCG migrated to a new electronic healthcare system in 2017, resulting in the antibiotic data not being available at the time of publication. There was a VRE outbreak in 2017, which would have allowed us to build these models on the 2017 outbreak and validate them on the 2018 outbreak. Once these data become available, this could be a future research opportunity.

Even though this study can determine if a patient were using antibiotics at a particular time, we could not distinguish between the types of antibiotics used. Some antibiotics target specific bacteria and can have a more significant effect on the risk of being colonised with VRE. A future research opportunity is to create antibiotics centrality measure for antibiotics targeting different bacteria.

The risk of VRE colonisation may differ between the types of hospital wards. For instance, patients admitted to an ICU may be more likely to be colonised with VRE than for general wards. We assumed that the difference in risk of VRE colonisation at the ward level could be explained by the number of patients, their antibiotics usage, and how patients in general and patients using 
antibiotics transition towards each ward using the centrality measures proposed in this study. For instance, the probability that at least one patient is colonised with VRE for a ward having a large number of patients using antibiotics and a relatively high probability that a patient using antibiotics may end up there compared to a general ward with low antibiotics usage. Even though the proposed models could predict VRE colonisation at the ward level, they may be further expanded to the patient level to test this assumption. This expansion will require additional patient data regarding demographics and comorbidities affecting the risk of VRE colonisation. A prediction model for VRE colonisation at the patient level using the proposed spatiotemporal centrality measures and patient-level data will also improve the efficiency of infection prevention specialists to control AMR in hospitals.

Even though the models focussed on predicting VRE colonisation at the ward level, the proposed spatiotemporal centrality measures may be used to generalise the models for other transmittable microbes in hospital environments. Future research may validate the relevance of these measures in a multicentre study using other outcome variables based upon, for example, carbapenem-resistant Enterobacteriaceae (CRE) or Clostridium difficile.

\section{Conclusion}

This study showed how the movements of patients inside hospitals and their use of antibiotics could predict the VRE colonisation of patients at the ward level. Two daily centrality measures were proposed to summarise the flow of patients and antibiotics at the ward level. A simple set of rules was produced which can be used to monitor the risk of VRE colonisation in hospital wards. A random forest ensemble model was compared with a decision tree model to improve the prediction performance at the cost of simplicity. An early warning system for VRE colonisation can be developed to test and further develop infection prevention plans and outbreak strategies using these results.

\begin{abstract}
Abbreviations
AMR: Antimicrobial resistance; AUC: Area under the curve; $\mathrm{Cl}$ : Confidence interval; CRE: Carbapenem-resistant Enterobacteriaceae; DG: Dynamic directed spatiotemporal graph; EHR: Electronic health record; HCW: Healthcare worker; ICU: Intensive care unit; MSE: Mean square error; NGS: Next generation sequencing; PCR: Polymerase chain reaction; PR: PageRank; RF: Random forest; ROC: Receiver operator characteristic; RSS: Residual sum of squares; UMCG: University Medical Center Groningen; VRE: Vancomycin-resistant Enterococcus.
\end{abstract}

\section{Acknowledgements}

S. (Simon) van der Pol from UMCG for making the with PCR test data available in a digestible manner.

\section{Authors' contributions}

$J M$ and $M$ contributed to the conceptualisation of the research. M, A, LMA and JEWC commented on the draft and final version of the paper. JM and $M$ contributed to editing and writing the draft and final version of the paper. JM performed the data analysis, statistical analysis and wrote the draft and final version of the paper. All authors read and approved the final manuscript.

\section{Funding}

This research was also supported by the INTERREG VA (202085) funded project EurHealth-1 Health (http://www.eurhealth1 health.eu), part of a Dutch-German cross-border network supported by the European Commission, the Dutch Ministry of Health, Welfare and Sport, the Ministry of Economy, Innovation, Digitalisation and Energy of the German Federal State of North RhineWestphalia and the Ministry for National and European Affairs and Regional Development of Lower Saxony.

\section{Availability of data and materials}

The data that support the findings of this study are available from UMCG but restrictions apply to the availability of these data, which were used under license for the current study, and so are not publicly available. Data are however available from the authors upon reasonable request and with permission of UMCG.

\section{Declarations}

\section{Ethics approval and consent to participate}

This study was approved by the Medical Ethics Review Board University Medical Centre (UMCG, METc 2019/409). Due to the retrospective and anonymous nature of the study, the need for informed consent was waived by the Medical Ethics Review Board University Medical Center. The study was carried out in accordance with relevant guidelines and regulations. In addition, the METC UMCG has determined that this study is not inside of the scope of the Research Involving Human Subjects Act (WMO).

\section{Consent for publication}

Not applicable.

\section{Competing interests}

The authors declare that they do not have any conflict of interest.

\section{Author details}

'Department of Psychology, Health and Technology/Center for eHealth Research and Disease Management, Faculty of Behavioural Sciences, University of Twente, Enschede, The Netherlands. ${ }^{2}$ Department of Earth Observation Science, Faculty of Geo-Information Science and Earth Observation (ITC), University of Twente, Enschede, The Netherlands. ${ }^{3}$ Department of Medical Microbiology, University of Groningen, University Medical Center Groningen, Groningen, The Netherlands.

Received: 31 August 2021 Accepted: 7 January 2022

Published online: 20 January 2022

\section{References}

1. O'Driscoll T, Crank CW. Vancomycin-resistant enterococcal infections: epidemiology, clinical manifestations, and optimal management. Infect Drug Resist. 2015;8:217-30.

2. Datta R, Juthani-Mehta M. Burden and management of multidrug-resistant organisms in palliative care. Palliative Care. 2017. https://doi.org/10. $1177 / 1178224217749233$.

3. Satilmis L, Vanhems P, Bénet T. Outbreaks of Vancomycin-resistant enterococci in hospital settings: a systematic review and calculation of the basic reproductive number. Infect Control Hosp Epidemiol. 2015;37(3):289-94.

4. Mutters NT, Mersch-Sundermann V, Mutters R, Brandt C, Schneider-Brachert W, Frank U. Control of the spread of vancomycin-resistant enterococci in hospitals: epidemiology and clinical relevance. Deutsches Arzteblatt Int. 2013;110(43):725-32.

5. García Martínez de Artola D, Castro B, Ramos MJ, Díaz Cuevas Z, Lakhwani S, Lecuona M. Outbreak of vancomycin-resistant enterococcus 
on a haematology ward: management and control. J Infect Prevent. 2017;18(3):149-53.

6. Blanco N, O'hara LM, Harris AD. Transmission pathways of multidrugresistant organisms in the hospital setting: a scoping review. Infect Control Hosp Epidemiol. 2019;40(4):447-56.

7. Frakking FNJ, Bril WS, Sinnige JC, van't Klooster JE, de Jong BAW, van Hannen EJ, et al. Recommendations for the successful control of a large outbreak of vancomycin-resistant Enterococcus faecium in a non-endemic hospital setting. J Hosp Infect. 2018;100(4):e216-25.

8. Drees M, Snydman DR, Schmid CH, Barefoot L, Hansjosten K, Vue PM, et al. Prior environmental contamination increases the risk of acquisition of vancomycin-resistant Enterococci. Clin Infect Dis. 2008;46(5):678-85.

9. Mcdermott H, Skally M, O'rourke J, Humphreys H, Fitzgerald-Hughes D. Vancomycin-resistant Enterococci (VRE) in the intensive care unit in a nonoutbreak setting: identification of potential reservoirs and epidemiological associations between patient and environmental VRE. Infect Control Hosp Epidemiol. 2018;39(1):40-5.

10. Huang SS, Datta R, Platt R. Risk of acquiring antibiotic-resistant bacteria from prior room occupants. JAMA Intern Med. 2006;166(18):1945-51.

11. Mitchell BG, Dancer SJ, Anderson M, Dehn E. Risk of organism acquisition from prior room occupants: a systematic review and meta-analysis. J Hosp Infect. 2015;91(3):211-7.

12. Ford CD, Lopansri BK, Gazdik MA, Webb B, Snow GL, Hoda D, et al. Room contamination, patient colonisation pressure, and the risk of vancomycinresistant Enterococcus colonisation on a unit dedicated to the treatment of hematologic malignancies and hematopoietic stem cell transplantation. Am J Infect Control. 2016:44(10):1110-5.

13. Pan SC, Wang JT, Chen YC, Chang YY, Chen ML, Chang SC. Incidence of and risk factors for infection or colonisation of vancomycin-resistant enterococci in patients in the intensive care unit. PLOS ONE. 2012;7(10):1-8.

14. Dik JWH, Hendrix R, Poelman R, Niesters HG, Postma MJ, Sinha B, et al. Measuring the impact of antimicrobial stewardship programs. Expert Rev Anti Infect Therapy. 2016;14(6):569-75.

15. Bush K, Barbosa H, Farooq S, Weisenthal SJ, Trayhan M, White RJ, et al. Inpatient mobility to predict hospital-onset Clostridium difficile: a network approach. BioRxiv. 2018;265.

16. Kaya A, Kaya SY, Balkan II, Bayramlar OF, Mete B, Saltoglu N, et al. Risk factors for development of vancomycin-resistant enterococcal bacteremia among VRE colonisers: a retrospective case control study. Wien Klin Wochenschr. 2021;133(9-10):478-83.

17. Rozenshtein P, Gionis A, Prakash BA, Vreeken J. Reconstructing an epidemic over time. Proc ACM SIGKDD Int Conf Knowl Discov Data Mining. 2016;13-17-Augu(IC):1835-44.

18. Gleich DF. PageRank beyond the web. SIAM Rev. 2015;57(3):321-63.

19. Breiman L, Friedman J, Stone CJ, Olshen RA. Classification and regression trees. Boca Raton: CRC Press; 1984.

20. Kingsford C, Salzberg SL. What are decision trees? Nat Biotechnol. 2008;26(9):1011-2.

21. Hanley JA, McNeil BJ. The meaning and use of the area under a receiver operating characteristic (ROC) curve. Radiology. 1982;143(1):29-36.

22. Team RC. R: a language and environment for statistical computing. 2019;3.

23. Csardi G, Nepusz T. The igraph software package for complex network research. InterJournal. 2006;Complex Systems:1695.

24. Paluszynska A, Biecek P, Jiang Y, Jiang MY. Package 'randomForestExplainer'. Explaining and visualizing random forests in terms of variable importance. 2017;

25. Liaw A, Wiener M. Classification and regression by randomForest. R News. 2002;2(3):18-22.

26. Wickham H, Averick M, Bryan J, Chang W, McGowan LD, François R, et al. Welcome to the Tidyverse. J Open Source Softw. 2019;4(43):1686.

\section{Publisher's Note}

Springer Nature remains neutral with regard to jurisdictional claims in published maps and institutional affiliations.

Ready to submit your research? Choose BMC and benefit from:

- fast, convenient online submission

- thorough peer review by experienced researchers in your field

- rapid publication on acceptance

- support for research data, including large and complex data types

- gold Open Access which fosters wider collaboration and increased citations

- maximum visibility for your research: over $100 \mathrm{M}$ website views per year

At BMC, research is always in progress.

Learn more biomedcentral.com/submissions 